\title{
Discovery of novel bioactive natural products from Streptomyces driven by a bottom-up approach
}

\author{
Jaka Widada ${ }^{1}$ \\ ${ }^{1}$ Department of Agricultural Microbiology, Universitas Gadjah Mada, Bulaksumur, Yoyakarta 55281, Indonesia
}

\begin{abstract}
Streptomyces strains are a very potential source for bioactive natural products of great interest in the pharmaceutical industry such as antibiotics, anticancer chemotherapy, immunosuppressants, etc. About two thirds of all known antibiotics are produced by actinomycetes, mostly by Streptomyces. However, in recent years, the chances of discovering new and bioactive natural products from Streptomyces have decreased significantly. In general, the selection of antibiotic- producing Streptomyces was done by using an antagonist to test a number of microbial pathogens so that only strains that had the inhibitory ability were forwarded for further investigation, while strains that did not have the ability were discarded. For our research group, it is also interesting to explore furtherStreptomyces strains that do not produce antifungal compounds in producing new bioactive natural products such as anticancer and anti-inflammatory. Our hypothesis is that the bioactive natural products produced from this strain will be safe if the compound is developed as a drug, because of its low cytotoxicity to non-target cells. The bottom-up approach through genome sequencing has provided access to a large number of BGC bioactive natural products embedded in the Streptomyces genome. In addition, metabolomics studies provide a portfolio of the entire metabolite producedfrom the strain of interest. Therefore, in this presentation, we will present a bottom- up approach to accelerate the discovery of bioactive natural products especially anticancer from Streptomyces sp. GMY01 isolated from the sediments of the southerncoast of Gunung Kidul, Yogyakarta by combining genomic mining and metabolomic approaches.
\end{abstract}

